\title{
Generative Replication and the Evolution of Complexity
}

\author{
Geoffrey M. Hodgson and Thorbjørn Knudsen
}

The Business School, University of Hertfordshire, De Havilland Campus, Hatfield, Hertfordshire AL10 9AB, UK g.m.hodgson@herts.ac.uk and

Strategic Organization Design Unit (SOD), Department of Marketing \& Management, University of Southern Denmark, Odense Campus, DK-5230, Odense M, Denmark

KEY WORDS: replication, replicators, information, complexity, automata

JEL classification: B52, D83

\begin{abstract}
This paper identifies generative replication as a form of replication which has the potential to enhance complexity in social and biological evolution, including the wondrous complexity in the biological world, and complex social institutions such as human language and business corporations. We draw inspiration from the literature on self-reproducing automata to clarify the notion of information transfer in replication processes. To enhance complexity, developmental instructions must be part of the information that is transmitted in replication. In addition to the established triple conditions of causality, similarity and information transfer, a generative replicator involves a conditional generative mechanism that can use signals from an environment and create developmental instructions. We develop a simple model, a one dimensional linear automaton that is consistent with our four proposed conditions for a generative replicator. We show that evolution within this model will indeed approach maximal complexity, but only if our four proposed conditions are not violated.
\end{abstract}




\section{Generative Replication and the Evolution of Complexity}

When does replication lead to greater complexity? As the origin of complex forms of organization is one of the most fundamental problems in science, this question is of considerable importance, in particular in the natural sciences. It is a more recent concern in the social sciences (Rosser, 2009) where much prior effort has been directed at understanding how agents are able to cope with complexity (Blau \& McKinley, 1979; Brown and Eisenhardt, 1997; McQuiston, 1989; Weiss, 1982). As recent contributors to the literature on complexity in the social sciences (Rosser, 2009), we believe that the question of origins and evolution of complex social institutions should be at the center of attention. ${ }^{1}$

The goal of this paper is to offer a novel perspective on replication processes that can potentially explain why evolution sometimes lead to dramatic gains in complexity - and sometimes leads nowhere. We explain what kind of replicating entities are required to increase the complexity of an evolving social or biological population. These special replicators are referred to as generative replicators. Examples include routines, and, of course, genes. We formulate four axioms that jointly lay out the necessary and sufficient conditions for the existence of generative replication. Our argument is supported by a formal model drawn from the field of automata studies (von Neumann, 1966; Sipper, 1998; Freitas and Merkle, 2004).

One of the triumphs of the modern theory of evolution is that it can explain the origins of very complex living things (Dawkins, 1986). A process characterized by the general Darwinian principles of variation, selection and inheritance (or replication) can over longer periods of time lead to amazing increases in complexity. The canonical example is the evolution of the human eye. Even if its complex design suggests the presence of a superior creative force, we know that the eye emerged because of accumulated gradual transformations over long periods of time.

Social evolution has led to increases in cultural complexity that can be compared to the evolution of the human eye. Through very long periods of time, we can observe the emergence of increasingly complex social institutions and organizations starting from the evolution of pre-linguistic culture, human language, and tribal customs, to writing and records, states and laws, and the institutionalization of science and technology. Each new transition in the evolution of culture has retained core features of prior developments and then added a new layer of complexity. Indeed, there seems to be no end to the increase in complexity in the social world.

The process of replication is central to explaining the increase of complexity that has happened during the course of social and economic evolution. Replication involves transfer of information caused by a source so that the copy is similar to the original. Many forms of replication have this signature, including copying with sound recorders, photocopiers, nests

\footnotetext{
1 The authors are particularly grateful to Michael Christensen, Sidney G. Winter and to participants at the workshop on "Transdiciplinary Perspectives on Economic Complexity" at James Madison University on 17 May 2008 for comments and discussions. We also thank Howard Aldrich, Markus Becker, Marion Blute, David Hull, Daniel A. Levinthal, Pavel Luksha, Joel Mokyr, Pavel Pelikan, Peter Richerson, Kim Sterelny, Nils Stieglitz, Viktor Vanberg, Ulrich Witt and an anonymous referee. This paper makes use of some material from Hodgson and Knudsen (in press). Thorbjørn Knudsen acknowledges support from the Danish Social Science Foundation (FSE).
} 
and burrows. But in order to increase complexity one more condition must be fulfilled. We argue that there must be a transfer of a construction mechanism that can create a new entity on the basis of a fairly simple set of instructions.

Rather than copying all the details of the fully scaled-up entity, the transfer of information can be compressed. Genes allow transfer of information in compressed form. A gene is a biological example of such a conditional generative mechanism. All such mechanisms have the potential to generate new copies conditional on the input signals they receive from their environment. Replicators that reliably transfer conditional generative mechanisms can enhance complexity, but replicators that are beset with copy error cannot. Since it is obviously important to identify replicators that can enhance complexity, we reserve the term "generative replicators" to describe them.

Habits and routines are the most obvious examples of generative replicators in the social context. A habit is a disposition to engage in previously adopted or acquired behavior triggered by an appropriate stimulus. Habits replicate indirectly, by means of their behavioral expressions. They can impel behavior that is followed by others, as a result of incentive or imitation. Eventually, the copied behavior becomes rooted in the habits of the follower, thus transmitting from individual to individual an imperfect copy of each habit. Routines are organizational dispositions made up of habits that are developed in an organizational context.

It is important to note that habits and routines are characterized as dispositions (or propensities) - they reliably map particular input signals onto particular behaviors. While the observable behavior is a signature of habits and routines, its core is the cognitive component that maps the reading of cues onto behavior. It is the possibility of replicating the underlying cognitive component that gives habits and routines status as generative replicators.

A concrete example of a persistent cognitive component of a routine is useful. The routine in question evolved in the military (Morison, 1966). Because of shortage in supplies of armaments during WWII, a piece of light artillery was hitched to trucks and then served as mobile coast defense. Even if the new device worked, the rapidity of fire was thought to be rather slow. Various experts looked into the matter and noticed a strange interruption each time the gun was fired. The crew simply ceased all activity and came to attention until the gun had been discharged. This routine considerably slowed down the rapidity of fire, but no one had a clue why it occurred. Eventually, an old colonel solved the riddle (Morrison, p. 17):

"Ah," he said when the performance was over, "I have it. They are holding the horses."

To be sure, it was a gun that had been used 60 years before in the Boer wars. At that time, it was important to hold the horses during gun fire, but by the 1940s the horses were long gone. Had the old colonel not been around, it is doubtful if anyone would have understood what happened. Even while the context had changed in dramatic ways, the underlying cognitive component of the routine had somehow replicated across generations of soldiers. The event of gun fire reliably triggered an observable behavioral trait among members of a crew that operated the gun many years after the horses were relieved from duty.

This example illustrates how routines can function as generative replicators. The gun firing routine had been reliably replicated through the regimen of drilling recruits on parade fields. During endless repetition of behavior, the cognitive component of the routine was also transferred, but had gradually faded beyond conscious awareness.

Generative replication is a natural construct for analyzing routines over the long term. By long term we refer to evolutionary dynamics that unfolds over decades and centuries. But our argument also has important implications for the typical empirical (case) studies of routines 
that span a few years or even shorter periods of time. We find that generative replication can involve two kinds of error that are both prominent in social and economic evolution. The first is copy error. Copy error changes the cognitive component of a routine so that input signals are associated with new forms of behavior. Within the context of our previous example, a copy error might associate the firing of a gun with all kinds of behavior. We find that anything but the smallest dose of this form of error is absolutely detrimental to the evolution of complexity. The second form or error is when the generative replicator misreads instructions. Read error changes behavior, but not the cognitive component. This kind of error erodes order in the observable trait pattern, but retains the underlying replicator. Without copying error, the cognitive component would reliably be replicated across time even with significant read error and notable disorder in observable patterns of behavior.

Our findings have far reaching implications for empirical research on routines (Becker, 2008). While observable patterns of behavior may indeed indicate the presence of a routine, it could just as well be hidden under a more disorderly and irregular behavior pattern. It is therefore time to discard any understanding of routines principally in terms of observable patterns of behavior. What we need instead is manipulation of experimental conditions that allow inference about the presence (or absence) of the underlying cognitive component of routines.

The remainder of this paper is organized as follows. In section two we connect to the literature on replicators and replication in order to examine widely used definitions of replication. Replication is usually defined in terms of the triple conditions of causality, similarity and information transfer. This triplet is necessary but insufficient in defining generative replication, which has the potential to enhance complexity. Section three draws from the literature on self-reproducing automata to strengthen the notion of information transfer in replication processes. Essentially, generative replication requires that developmental instructions are part of the information that is transmitted in replication.

Section four establishes the concept of generative replication. In addition to the triple conditions of causality, similarity and information transfer, we adopt a new condition that defines a generative replicator as containing conditional generative mechanism that can turn input signals from an environment into developmental instructions. Section five examines the conjecture that generative replication has the potential to enhance complexity. We develop a simple model, a one dimensional linear automaton that is consistent with our four proposed conditions for a possible generative replicator. This model is particularly appropriate for social evolution. Section six extends our model to a simple selection dynamics. Our results provide strong and unambiguous support for the complexity conjecture. Section seven concludes the paper.

\section{Replicators and Replication}

Dawkins (1976) sees replicators as having longevity, fecundity and fidelity. Dawkins (1982, 2004) upholds that replication involves a degree of genotypic copying fidelity generally sufficient for genotypic errors and mutations to be passed on to successive generations. $\mathrm{He}$ identified both genes and "memes" as replicators. There is ongoing controversy on what a meme is, and little progress in identifying the equivalent to the "genetics" of memetic replication. David Hull (1988, p. 408) defines a replicator as "an entity that passes on its structure largely intact in successive replications." A key question here is what structures are significant and why. 
Kim Sterelny et al. (1996, p. 395) offer a more elaborate definition of a replicator. They propose that if $\mathrm{B}$ is a copy of $\mathrm{A}$, and $\mathrm{B}$ is produced through a process of replication, then " $\mathrm{A}$ plays a causal role in the production of $\mathrm{B}$ " and "B carries information about $\mathrm{A}$ in virtue of being relevantly similar to A. This similarity is often functional: B has the same, or similar, functional capacities to A". Their definition emphasizes the three key points of causal implication, similarity and information transfer, which are central to most subsequent definitions of replication.

But with these three elements Sterelny et al. admit a very wide class of entities, where nonorganisms such as bird nests and animal burrows qualify as replicators. But the causal role that one nest or burrow plays in the production of another is highly limited. If nests and burrows are to be admitted as replicators, then their causality condition has to be interpreted in very weak terms. The causal link is merely that each replicator is a practice model for its successors. Arguably the causality condition has to be coupled with other stipulations that make it less loose and more meaningful. Our definition below of "generative replicators" excludes nests, burrows, and many human artifacts. But it includes habits, routines and conventions.

In another development of the replicator concept, Peter Godfrey-Smith (2000, p. 405) emphasizes that replication involves "two main elements, a resemblance between copy and copied, and some suitable causal relation linking the copy to the copied." He is notably skeptical of versions of cultural evolution based on the meme.

Dan Sperber (2000, p. 169) refines the information transfer condition in the following manner: "The process that generates B must obtain the information that makes B similar to A from A. ... B must inherit from A the properties that make it relevantly similar to A." $\mathrm{He}$ argues that many cases of so-called memetic replication do not count as replication, principally because his information transfer condition is violated. Hence the "grand project of memetics ... is misguided" (p. 173). ${ }^{2}$

A problem is that the three conditions of causality, similarity and information transfer still cover a broad class of copying processes. They would also apply to technologies such as photocopying, and the copying of data on magnetic media or on compact disks. The original is causally implicated in the production of the copy, in the weak sense that without the original the copy could not exist. Sperber (2000) upholds that the "information transfer" condition is satisfied in such cases because the "information" in the copy originates from the original.

Sperber's refined information transfer condition takes us in the right direction, but it is insufficiently precise (Hodgson and Knudsen, in press). It depends crucially on what is meant by "information" and what properties have to be inherited to constitute true replication. A critical issue pertains to the replication of a copying mechanism, or the absence of such processes. We consider this in the discussion of self-reproducing automata in the next section.

\footnotetext{
2 Aunger (2002) adds a fourth criterion of "duplication", meaning that replication of one entity gives rise to two or more. We are unconvinced of the utility of this condition. The critical issue in understanding the process of replication is the nature and function of the structure that is passed on, including any information that it might hold. It is not whether the replicator is lucky to survive the process of replication, leading or not to the coexistence of multiple copies.
} 


\section{Inspiration from Self-Reproducing Automata}

In lectures posthumously edited and published as a book, John von Neumann (1966) considered when automata would be capable of generating novelty and additional complexity. Von Neumann (1966, p. 80) distinguished between the copying of entities that synthesized to produce higher degrees of complexity, and copying that reduced overall complexity:

There is thus this completely decisive property of complexity, that there exists a critical size below which the process of synthesis is degenerative, but above which the phenomenon of synthesis, if properly arranged, can become explosive, in other words, where synthesis of automata can proceed in such a manner that each automaton will produce other automata which are more complex and of higher potentialities than itself.

Following von Neumann, we propose that generative replication should be confined to cases with the potential to increase complexity. This excludes sound recordings, photocopies, nests and burrows. Such copies have no more potential to enhance complexity than their predecessors. To increase potential complexity, copies must eventually be capable of producing novel additional components, or performing novel operations in response to new environmental conditions and input signals.

Von Neumann (1966) examined the properties that a self-reproducing automaton must possess to make it capable of producing other automata. These include: instructions that describe the structure and processes of an automaton, a copying unit that is capable of reading and copying instructions into a new automaton and translating them as directions for a production unit, a production unit that builds a new automaton, and some mechanism of coordination between these elements. His research on this topic has influenced robotics and computer science (Sipper, 1998; Luksha, 2003).

However, biological replication lacks the abstract properties of self-reproducing automata outlined by von Neumann. In particular, as developmental systems theorists emphasize, genes themselves do not carry enough "information" to describe or generate a new organism. Although the genes play a crucial role, the development of the organism depends additionally not only on environmental stimuli, but also on cellular structures inherited alongside the genes. Consequently, neither genes nor organisms constitute a complete "production unit" for new organisms. ${ }^{3}$

Often the information transferred within the replication process is insufficient to generate a copy, and an additional informational input is required. Nevertheless, some vital generative information can be passed on in replication. The problem is to identify the information with the potential enhance complexity.

The theory of self-reproducing automata considers the potential to enhance complexity and the developmental instructions that are part of the information that is transmitted on replication. These two inspirations lead us to the concept of generative replication. A generative replicator is a material entity that is responsive to environmental stimuli. There is at least one signal that can cause a non-degenerative response from the replicator. Such a

\footnotetext{
3 Pigliucci and Kaplan (2006, p. 80) eschew the replicator concept because: "Replication itself demands, in general, the organism's ability to interact. Genomes do not 'self-replicate." We agree with this statement but the rejection of the replicator concept does not follow. As elaborated here, prominent recent definitions of the replicator do not involve any "self-replication" that is independent of any environmental influences or context. Our concept of generative replication explicitly acknowledges the dependence on other structures and environmental inputs.
} 
response consists of further instructions or signals to the interactor, which guide its development. It is non-degenerative in the sense that it leads to outcomes that are conducive to the survival of the replicator and the information it carries.

\section{Defining the Generative Replicator}

Previously we noted three established definitional features for a replicator, namely causal implication, similarity and information transfer. We refine these as follows:

1. Causal implication: The source must be causally involved in the production of the copy, at least in the sense that without the source the particular copy would not be created.

2. Similarity: The copy must also possess the capacity to replicate. The conditional generative mechanisms (defined below) in the copy must be similar to those in the source. Errors or mutations in these mechanisms must also be copied with some degree of fidelity.

3. Information transfer: The process that generates the copy must obtain the conditional generative mechanisms that make the copy similar to its source from that same source.

Note how the causality condition is clarified. The enhanced similarity condition requires that similarity must apply to the conditional generative mechanisms, as emphasized by Dawkins (2004). A related refinement appears in the information transfer condition. On the basis of our discussion of self-reproducing automata, we adopt a fourth proposition in the definition of a generative replicator:

4. Conditional generative mechanisms: Generative replicators are material entities that embody construction mechanisms (or "programs") that can be energized by input signals, containing information about a particular environment. These mechanisms generate further instructions from the generative replicator to the interactor, to guide its development. ${ }^{4}$

Together these conditions preserve the spirit of Hull's (1988, p. 408) early definition of a replicator as "an entity that passes on its structure". An entity that satisfies all these four conditions is described as a generative replicator.

One of the problems in defining the replicator concept is what exactly "information" means. Our specification helps to fill this gap. The concept of information here does not necessarily carry interpretations or meanings in the same way as the information communicated by humans. It is information in a cruder sense of a code or signal, as stored and manipulated by computers and present in the DNA. For Claude Shannon and Warren Weaver (1949) a message has "information content" when its receipt can cause some action. For us,

\footnotetext{
${ }^{4}$ External influences that produce outcomes unfavorable to the survival of the replicator or interactor are not described as input signals but as destructive forces. The distinction between internal and external influences obviously requires a demarcation of replicators and interactors against their environment. An interactor is a relatively cohesive and durable entity with effective boundaries between itself and its surrounding environment including other entities. This means that the internal relations between its component parts are generally more significant, dense and durable than the relations between the entity and elements in its external environment. The existence of replicators further depends on the existence of their host interactors; if the interactor expires, so does the replicator that it hosts. We have clarified these conditions elsewhere (Hodgson \& Knudsen, 2004b).
} 
the "information" involved consists of signals with the potential to trigger generative mechanisms that guide the production of further replicators or the development of interactors.

By contrast, ideas involve meanings and interpretations. Furthermore, our added condition insists on the materiality of the generative replicator, hence ideas as such are not generative replicators. By contrast, habits and routines may qualify as generative replicators (Hodgson \& Knudsen 2008).

Our concept of "conditional generative mechanism" is close to what Ernst Mayr (1974, 1988) describes as a "program". Mayr (1988, p. 48) regards a program as something embodied in a material substrate that gives rise to goal-driven (or "telenomic") behavior, and hence is "consistent with a causal explanation". The closest social science equivalent is the notion of "programs" (March and Simon, 1958) developed in the behavioral research program.

Nests, burrows and photocopies are not generative replicators. None of these entities is capable of receiving and emitting signals that lead to the development of the interactor. The footnote to condition (4) establishes that destructive or degenerative environmental influences do not count as signals. Having made this exclusion, nests, burrows and photocopies have no conditional mechanisms to guide the development of the interactor. They cannot enhance complexity through replication and hence they are not generative replicators.

\section{Generative Replication and Complexity}

Both biological and social evolution exhibit vast complexity (Buss, 1987; Dawkins, 1986; Maynard Smith and Szathmáry, 1995). The question whether or not evolution necessarily leads to greater complexity has attracted much interest and debate (Adami, 2002; Adami et al., 2000; Bennett, 1995; Gould, 1996; McShea, 1996). The jury is still hung on the verdict.

The disagreement not only concerns a possible positive trend of (biological) complexity caused by evolution, but also what definitions and measures of complexity to use when this claim is assessed (Adami et al., 2000). A recent review of many definitions and measures of complexity concluded that most of these have drawbacks of either a conceptual or a practical nature when used to capture the complexity of evolving entities (Adami, 2002).

Consistent with information theory, Christoph Adami (2002) finds that the essence of complexity for an evolving entity is the amount of information that it stores, in its genome, about the environment in which it evolves. Adami (2002: 1087) defines the physical complexity of a sequence of symbols as follows:

The physical complexity of a sequence refers to the amount of information that is stored in that sequence about a particular environment. For a genome, this environment is the one in which it replicates and in which its host lives, a concept roughly equivalent to what we call a niche. Information ... is always about something. Consequently, a sequence may embody information about one environment (niche) while being essentially random with respect to another. This makes the measure relative, or conditional on the environment, and it is precisely this feature that brings a number of important observations that are incompatible with a universal increase in complexity in line with a law of increasing physical complexity.

This definition conceptualizes complexity as information relative, or conditional on a particular environment (or niche) within which the entity evolves. It further implies that 
information is obtained from input signals that are actually recorded in a sequence stored in a generative replicator (genome), which in turn requires a distinction between replicators and interactors. These aspects of physical complexity are all captured by our notion that the generative replicator is a material entity that embodies conditional generative mechanisms that can be energized by external signals (a sequence of information about a particular environment).

Our definition of a conditional generative mechanism further highlights that the information stored in a generative replicator is energized by external signals to guide the development of the interacting entity, and disregards signals which lead to outcomes that are generally unfavorable to its survival. Our definition focuses on how a new replicator obtains its sequence of information about a particular environment.

A critical part of our argument is the complexity conjecture:

Within complex population systems, evolution will approach the maximal level of complexity if and only if all four conditions in the definition of a generative replicator are met (conditional generative mechanism, causality, similarity and information transfer).

To support this conjecture, we develop a simple model - a one dimensional linear automaton that is consistent with our four proposed conditions for a possible generative replicator. Our model is particularly applicable to social evolution.

\section{Model}

The model we develop below is simple and heuristic. Some of its features make it applicable to social but not biological evolution. We use the classical one dimensional (1D) linear automaton with two states (Molofsky, 1994; Wolfram, 1984, 2002) because it is well understood and offers the simplest testbed for studying replication. The simple cellular automaton characterizes an evolving population of entities. A binary ring represents a constant population of $n$ interacting entities at a given time step. The evolution of a population is a discrete dynamics captured by successive binary rings. The cells in the binary ring represent trait values of individual entities; each entity has only one trait with two states, zero or one. The actual trait value of an entity and its two immediate neighbors jointly determine the probability with which the entity replicates the instructions that led to the development of that trait value.

The development of an entity's trait value depends on the instructions carried in its generative replicator. At each time step, there is replication of a rule (an information sequence comprising the generative replicator) and a subsequent process of development where the generative replicator is energized by input signals from its two immediate neighbors. The outcome of the development process is a new individual entity whose trait value depends on the way the generative replicator reads and codes input signals from its two neighbors.

As an example, consider Figure 1 below where a particular generative replicator reads an input signal consisting of three components: the state of the neighbor to the left, the state of the entity itself (middle), and the state of the neighbor to the right. ${ }^{5}$

\footnotetext{
5 The number (e.g. 126) characterizing a replicator is the decimal equivalent to the binary sequence ' 01111110 ' for which it codes (see Figure 1).
} 


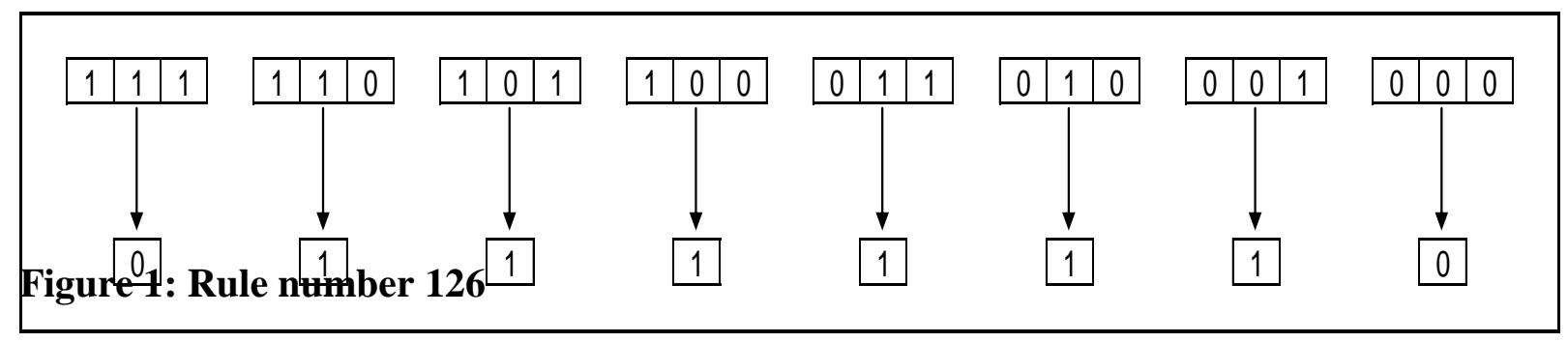

Prior research on self-replicating automata (Molofsky, 1994; Wolfram, 1984, 2002) has studied emergent properties when applying deterministic rules (e.g. generative replicator 126 shown in Figure 1) that read input signals without error and replicates without error.

Consider how the automaton in Figure 1 works. It is a testbed for a single replicator called rule 126. This replicator generates trait values conditional on input signals. The input signal to the replicator is the current state of the (infant) entity itself and of its two neighbors (can easily be generalized). The first row of Figure 1 shows the input signals consisting of three binary numbers (zeros or ones). The number in the middle is the state of the entity itself. The two other numbers represent the state of the immediate neighbors. The developmental process of generating a new trait value is represented by the arrow in Figure 1.

After the developmental process is completed, the replicator is copied and the process repeats itself. The copying of the replicator involves the possibly error-prone process of reproducing the map from input signals to trait values. A replication error would result in a new map (for example rule 255 which produces trait values of 1 for any input signal).

The automaton contains a number of interacting entities. Each entity works exactly as described here. In its most simple form, we place a single replicator such as rule 126 in the automaton and study its evolution. We expose the replicator population in the automaton to two different kinds of error: 1) error in reading input signals and 2) copy error. The effect of error in reading input signals is shown in Figure 2. This kind of error erodes order in the observable trait pattern, but retains the underlying replicator. Without copying error, then rule 126 would be reliably replicated across time. In different terminology, it would have maximal fitness.

When we expose a replicator population to reading error, there is a loss of order, but no loss of fitness. This means that the replicator population has the potential to restore order. By contrast, when we expose a replicator population to copy error, there is both a loss of fitness as well as a loss of order. Copy error will therefore cause an irreversible loss of order; reading error will not.

Compared with prior research, we extend the use of the 1D automaton in a number of ways. First, we consider a population of emergent generative replicators. We start from a soup of random generative replicator sequences. Rather than starting from replicators as the one shown in Figure 1, we begin with replicators that code for trait values in an entirely random way. These replicators have the potential to evolve into replicators that work as rule 126 . Whether this will happen depends on the conditions that the replicator population is exposed to. 
Rule126, Error reading input signal=0

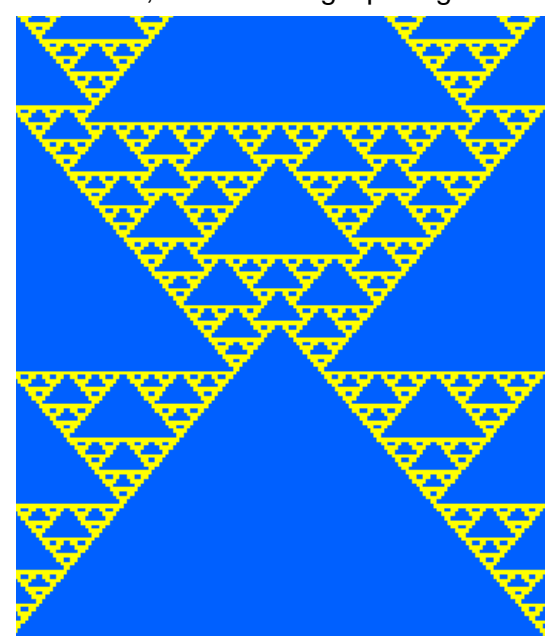

Rule126, Error reading input signal=0.05

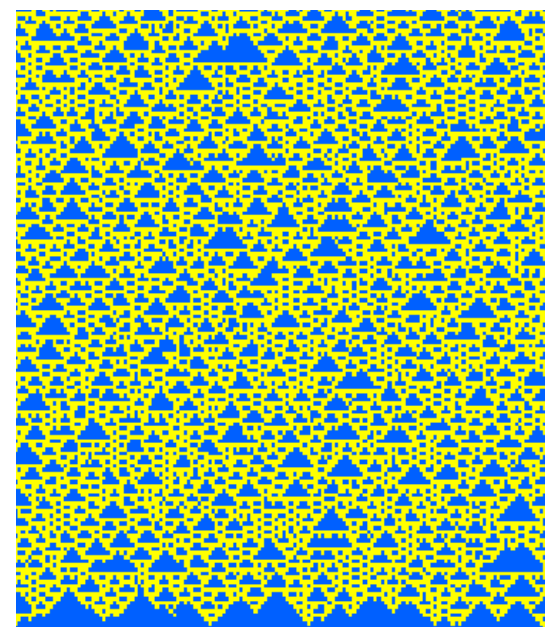

Rule126, Error reading input signal $=0.005$

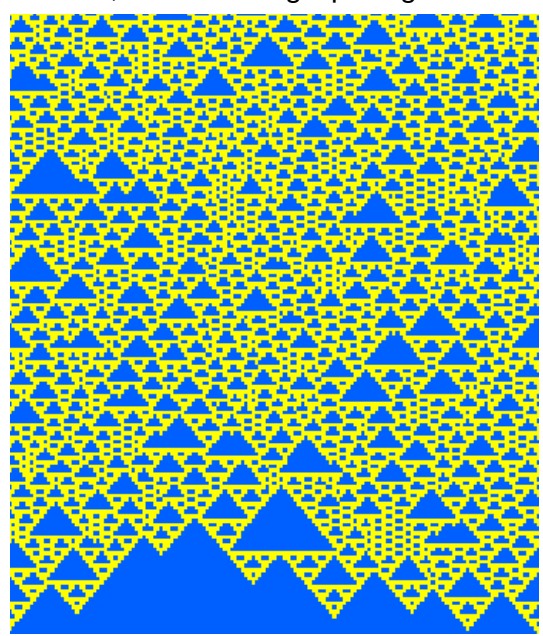

Rule126, Error reading input signal $=0.5$

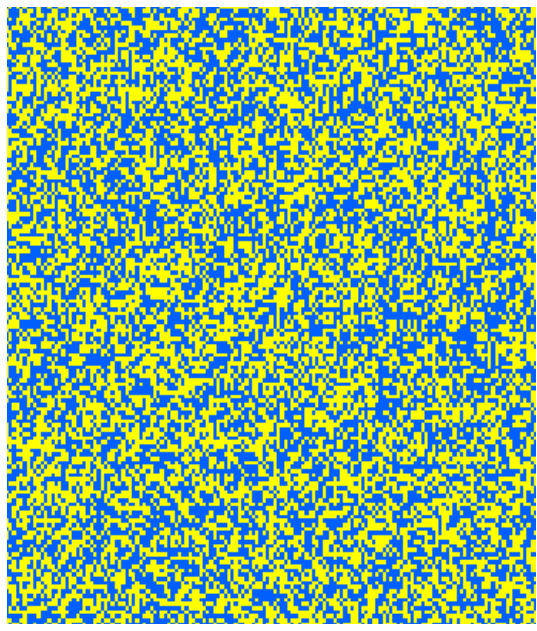

Figure 2: The expression of generative replicator 126 with different levels of error in reading input signals (perfect replication). Input error introduces noise, but does not necessarily decrease complexity.

Under some conditions, evolution will lead to the emergence of order. Thus, a number of different generative replicators may emerge from an initial stochastic soup. Our main argument relates to the conditions that favor the evolution of generative replicators. If all four of our conditions are met, we suppose that a significant number of generative replicators will emerge, thus giving rise to an increase in complexity towards some maximal level. 
Second, we consider the possibility of error in reading input signals (see Figure 2 below). Such error does not violate our four conditions defining a generative replicator. Also in this case, we expect complexity to approach the maximal level. In reality, there also could be development errors, where the input data is read correctly but mistakes are made in its enaction in the development of the entity. In terms of the complexity conjecture, the difference between reading and development error is less important than their differences with copy error. In order to reduce unnecessary complications, we do not make a distinction between reading and development errors in the models presented here (could easily be done).

Third, we also expose the conditional generative mechanism to error that violates our first condition defining a generative replicator. In consequence, complexity should not approach the maximal level (defined below) in the presence of replication error.

Overall, we are using the 1D automaton to provide an example that is consistent with the complexity conjecture. Our version of the 1D automaton is a useful test-bed for the study of an evolving population of generative replicators that are subject to either copy error or development error.

An eight step description of our elaborated version of the 1D cellular automaton is provided below. In order to test the complexity conjecture in the context of the $1 \mathrm{D}$ automaton, we examined four models. Two of these models violate our condition one, defining a generative replicator. Consequently, we expect that these two models also violate the complexity conjecture. By contrast, the two models that do not violate our definition of a generative replicator are expected to produce results that are consistent with the complexity conjecture.

\section{Eight step specification of the extended 1D two-state cellular automaton:}

1) The generative replicator is a sequence of eight components $\left(p_{1} \ldots p_{8}\right)$ that generate responses to input signals.

2) The eight components of a generative replicator's sequence are conditional probabilities $\left(p_{1} \ldots p_{8}\right)$ according to which a particular binary state (zero or one) will be realized in response to particular input signals. The realization of such a binary state represents the development of the trait of an entity (the realized trait value is either zero or one).

3) The generative replicator reads three input signals comprising the current state of the local environment (two neighbors) as well as the current state of the entity itself. There are eight possible combinations of input signals, each energizing a distinct component $\left(p_{i}, i=1 \ldots 8\right)$ of the generative replicator sequence.

4) In the first time step, the values of each entity's generative replicator components $\left(p_{1} \ldots p_{8}\right)$ are drawn from a uniform distribution. Subsequently the replicator components can vary because of replication error or when replacements occur as a consequence of the selection dynamics.

5) With probability $\varepsilon_{R}$, the generative replicator makes an error in reading the input signals. If this occurs, the interactor develops the reverse of the trait it would have developed in the absence of error, e.g. a zero instead of a one.

6) The generative replicator is copied. That is, the map from input signals to trait values is reproduced. The copy is preserved but not the original. 
7) There is a level of replication error $\varepsilon_{C}$. Replication error perturbs the replicator sequence $\left(p_{1} \ldots p_{8}\right)$ with Gaussian noise (mean zero and standard deviation $\left.\varepsilon_{\mathrm{C}}\right)$. For example, when a perturbation occurs, a replicator component with a value of $p_{1}=$ 0.17 can mutate and get a new value of 0.21 . The parameter $\varepsilon_{C}$ scales the second moment of the Gaussian noise distribution. It is set sufficiently low so that the replicator sequence $\left(p_{1} \ldots p_{8}\right)$ is bounded within the unit interval. In the unlikely event that a perturbation assigns a value outside the unit interval, it is replaced with a random draw from a uniform distribution in the unit interval.

Our main results on the evolution of complexity are quite robust to using alternative specifications. ${ }^{6}$

\section{Measuring complexity}

Following Adami (2002) and others, we conceptualize complexity as the information a generative replicator sequence stores about its environment. This is useful because it allows us to capture the informational property of a generative replicator in terms of the well understood measure of Shannon entropy from information theory. Measuring complexity as an instance of entropy is now well-established and we follow prior research (Adami et al, 2000; Adami 2002; Davis, 1979, 1994) in defining a suitable complexity measure.

For our automaton, population level complexity is the difference between the unconditional entropy of an environment, $H_{\max }$, captured by a string of length $L$ (the number of replicating entities) and the entropy of a set of generative replicator sequences operating within this environment.

The entropy of a population of generative replicators $X$, coding for a sequence of entities, whose traits $(0 / 1)$ occur with probabilities $p_{i}$ within a particular environment $E$, is denoted by the symbol $H(X)$. The theoretical measure of physical complexity, $C$, of a population of replicating entities is the information that the generative replicator sequences $X$ contain about the environment $E$ :

$$
C=H_{\max }-H(X)=L+\Sigma p_{i} \log p_{i}
$$

The unconditional entropy is a measure of potential knowledge in terms of how much information the sequence, $L$, could possibly hold, and thus quantifies the (probabilistic) uncertainty of the information contained in this sequence (Adami, 2002). In order to estimate the physical complexity of the population, we obtain the substitution probabilities at each site, i.e. the probabilities that a generative replicator component coding for a 1(0) would instead result in a $0(1)$. That is, the substitution probabilities are the probabilities that a mutation will occur at a specific site (location in the string capturing the interacting entities). In this manner, we obtain the entropy at site $j$ :

$$
H(j)=-\Sigma p_{i}(j) \log p_{i}(j)
$$

The conditional entropy of a population $H(X)$ is approximated by summing over the L sites:

$$
H(X)=-\Sigma H(j)
$$

\footnotetext{
6 We have obtained similar results with a specification that used a reinforcement function rather than a selection dynamics. The reinforcement function was a classical habit function (Hull, 1943) where habituation is characterized in terms of an exponentially weighted average of past actions (e.g. Carroll, Overland \& Weil, 2000; Pollak, 1977).
} 
Thus, the approximate physical complexity is measured as:

$$
C_{a p p}=L-H(X),
$$

with $H(X)$ approximated by the substitution probabilities from equation (3).

The intuition underlying this measure is that complexity can be captured by the information that a generative replicator holds about an environment.

\section{Generative replication and selection}

One of our main arguments is that copy error and read error have different outcomes concerning the development of complexity. Why is this so? Consider a process of repeated replication with input signals, the development of interactors and the copying of generative replicators. Assume that there is no alteration of stored information in any individual generative replicator, implying no Lamarckian inheritance. In a fixed selection environment, these generative replicators would tend to develop traits clustered around a peak in a fixed fitness landscape. (Changing environments and variable or "dancing" fitness landscapes are excluded for simplicity, not because they would undermine our argument.)

Reading (and developmental) errors cause the population to disperse from its original position in the fitness landscape. However, if copy errors are zero, and the probabilities of reading and development errors remain constant, then the trend of overall dispersion will not increase through time as repeated replication occurs. The effects of reading or development errors are not cumulative in this model. Information content is preserved through the faithful copying of the generative replicator.

Re-start the process but assume that the probability of a copy error is positive. The population of generative replicators is again clustered around a peak in a fixed fitness landscape. If a generative replicator is close to the fitness peak, then copy error is likely to move it away from that peak. There will be no reliable mechanism to tie it to its previous position. A process of drift will occur and the overall dispersion in the population will increase. Hence the effects of copy error are cumulative. The entropy $H(X)$ of the population will increase and overall complexity will decline as a result. ${ }^{7}$

To further substantiate the proposition that the capacity to increase complexity depends critically on generative replication with low copy errors, we conducted a number of simulation experiments that added a simple selection dynamics to the above models.

Intuitively, selection is a replacement of a replicator with low fitness. We define fitness in terms of trait stability so that replicators that code for more stable traits have lower replacement probability. By contrast, replicators that code for less stable traits have higher replacement probability. When a replacement occurs, a replicator is eliminated and a new replicator is inserted at the location it previously occupied. The new replicator is generated in exactly the same way as described under step 4 in the model specification, i.e. each of the new replicator's components $\left(p_{1} \ldots p_{8}\right)$ are drawn at random from a uniform distribution.

\footnotetext{
7 Note that, if it did exist, "Lamarckian" inheritance could have similarly negative cumulative effect. Lamarckism upholds that the acquired characteristics of the interactor may affect the replicator, leading to the inheritance of these acquired characteristics. In biology, Lamarckian inheritance is widely ruled out because of the Weismann barrier. Although social evolution is widely described as Lamarckian, the meaning and relevance of the label is contested (Hodgson and Knudsen, 2006).
} 
Our model allows for independent variation of copy error and read error. If copy error is more than minimal, then the fourth of the above conditions defining a generative replicator (regarding conditional generative mechanisms) is violated. But tolerably high levels of read error are consistent with generative replication. Accordingly, we expect complexity to be increased as long as copy error remains low.

In each simulation, the fitness peak is defined in terms of a randomly generated (binary) fitness string of length $L=100$. The peak fitness string and the string representing the population are both binary strings of length $L$ and the difference between the two strings can be measured in terms of the Hamming Distance, i.e. the number of positions for which the corresponding values are different. This is population fitness component, $W(p)$.

Each entity in the population is subject to an individual fitness pressure defined in terms of trait stability; the more stable a trait is across time the higher the fitness. ${ }^{8}$ The individual fitness component $W(i)$ is defined as:

$$
W(i)=\mathrm{e}^{(\mathrm{r} / \tau) / \sum(\mathrm{r} / \tau)},
$$

where $\tau$ is a sensitivity parameter (this definition applies a widely used simplification of the Boltzmann distribution). The lower is $\tau$, the more important is relative variation in fitness components (at the limit when $\tau$ goes to zero, only one entity is fit). We set $\tau=0.05$ in all simulations reported here. ${ }^{9}$

Using a composition of population and individual fitness components, individual replacement probabilities, $P_{r}(i)$, are defined in the following way:

$$
P_{r}(i)=W(i) x W(p) x S
$$

where $S$ is a scale parameter regulating the selection pressure, $W(i)$ individual fitness and $W(p)$ is population fitness. ${ }^{10}$ All simulations reported here are obtained with selection pressure $S=1$.

We exercised each of the four models with a population of 100 replicating entities captured in a binary string of length $L=100$, arranged in a ring. The unconditional entropy of the environment is therefore, $L=100$. Interaction and replication occurred as described above. Interaction energizes the generative replicator so it reads three input signals comprising the current state of the local environment (two neighbors) as well as the current state of the interactor itself.

Our experiments comprised an evolution of 10,000 time steps. At each of the 10,000 time steps, we obtained the level of approximate physical complexity, $C_{a p p}$, according to equation (4). The maximal level of approximate physical complexity $C_{\max }=100$ characterizes a situation with no mutations, i.e. $H(X)=0$. We examined five levels of read error $\left(\varepsilon_{R}=0,0.01\right.$,

\footnotetext{
${ }^{8}$ We prefer to define fitness components from trait values. However, an alternative specification with selection directly on genetic components gives qualitatively similar (and numerically stronger) results. See Hodgson and Knudsen (in press) for further definition and discussion of the fitness concept in socio-economic evolution.

${ }^{9}$ Our results are robust to changes in this value.

10 The fitness function in equation 5 is motivated by the Price equation. The population fitness component, $W(p)$, captures a direct selection effect and the individual fitness component $W(i)$ captures an across generation transmission effect (a transmission of behavioural traits at the individual level). As we know from the recursive expansion of the Price equation, the transmission effect can be decomposed into direct selection effects at lower levels. An alternative specification where individual fitness components capture a direct selection effect on genetic components gives qualitatively similar (and numerically stronger) results.
} 
$0.05,0.10,0.50)$, five levels of copy error $\left(\varepsilon_{\mathrm{C}}=0,0.01,0.05,0.10,0.50\right)$, and then obtained 10 samples for each point in the configuration space. ${ }^{11}$

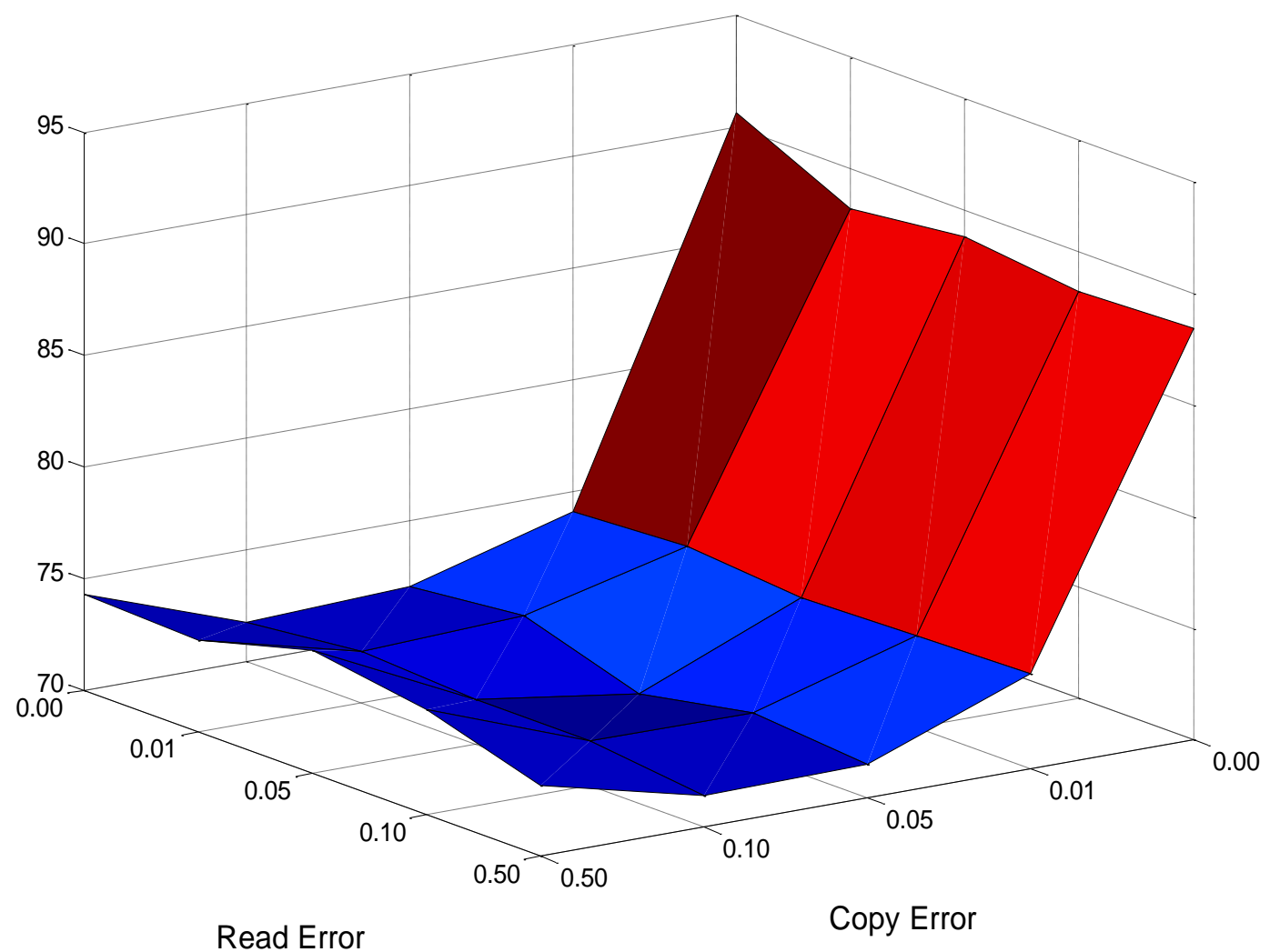

Figure 3: Complexity at time $T(=10,000)$ for five levels of read error and five independent levels of copy error. Based on averages of 10 samples, and 100 entities. Maximal complexity, $C_{\max }=100$.

Figure 3, shows that when there is neither copy error, nor read error the maximal level of complexity is approached $\left(C_{\max }=100\right)$ at time $\mathrm{T}=10000$. More interestingly, as long as copy error remains zero, thus protecting the generative replicators from harmful noise, very nearly obtains the same result even though the error in reading input signals becomes very high $\left(\varepsilon_{\mathrm{R}}=\right.$ $0.5)$. By contrast, with any level of copy error above zero, maximal complexity is not reached at $\mathrm{T}=10000$, and it never will, even when the replication error is low $\left(\varepsilon_{R}=0.01\right)$.

Runs with zero copy error approach maximal complexity no matter what the level of read error. By contrast, runs with positive copy error will never reach such high levels of complexity because copy error thwarts the approach to complexity. This result is very strong support for our conjecture that evolution will approach the maximal level of complexity if and only if all of the four conditions defining a generative replicator are met (conditional generative mechanism, causality, similarity and information transfer). As Figure 3 shows,

\footnotetext{
11 The standard deviations are low enough to make the results statistically significant for any pairwise comparison of treatments.
} 
copy error strongly dominates read error. The rise in complexity above the plateau of low complexity values only happens if copy error is zero.

Is it really so surprising that we see the evolutionary dynamics approach maximum complexity if there is no copy error? Since we are using an information-theoretic measure of complexity, this result tells us that an evolving replicator population looses track of its environment if there is copy error. This result is consistent with much prior research, and therefore unsurprising. What is more surprising is that we establish our result starting from a population of entities that have the potential to become replicators. Under some conditions, this potential is realized. Under other conditions, evolution leads nowhere. Our results thereby address a fundamental problem of identifying conditions that enable a population of entities to become a population of replicators. A further advantage of our approach is that we are able to establish that read error is fairly unproblematic. As we point out in the conclusion, this has important implications for our understanding and further study of selection arguments in economics.

Overall, the simple selection dynamics situated in the one dimensional linear automaton supported our complexity conjecture as well as the four proposed conditions for a possible generative replicator. We showed that a complex population system may approach the maximal level of complexity if only if all four conditions in the definition of a generative replicator are met. The gain in complexity is accompanied by an increase in fitness. That is, the dynamics settles more narrowly around the fitness peak if our four proposed conditions are not violated (the correlation between complexity and proximity to the fitness peak is 0.95 and significant at the 0.05 level).

\section{Conclusion}

This paper identifies a form of replication which has the potential to enhance complexity in social and biological evolution. Our finding points to a sharper explanation of processes where the accumulation of small changes can lead to improbable outcomes, including the wondrous complexity of the natural and the human social worlds.

One of the problems in defining the replicator concept, and of specifying a general Darwinian evolutionary process, is what exactly "information" means. Our specification helps to fill this gap. The concept of information used here does not necessarily carry interpretations or meanings in the same way as the information communicated by humans. For us, the "information" involved consists of signals with the potential to trigger generative mechanisms that guide the production of further replicators or the development of interactors.

Previous literature on replicators identifies the triple conditions of causality, similarity and information transfer. To define a generative replicator we adopt a fourth condition of a conditional generative mechanism; this is a material entity that embodies mechanisms that can turn input signals from an environment into developmental instructions. Essentially, generative replication has the potential to enhance complexity, which in turn requires that developmental instructions are part of the information that is transmitted in replication. This argument is more precisely expressed in our complexity conjecture: within complex population systems, evolution will approach the maximal level of complexity only if all four proposed conditions in defining a generative replicator are met (conditional generative mechanism, causality, similarity and information transfer). 
According to the complexity conjecture, different kinds of error should have very different impacts on the evolution of complexity. In particular, copy error that violates generative replication should inhibit the emergence of complexity when compared to read and development error. Notably, read and development error interfere with the reading of input signals from the environment and with developmental instructions, but these error forms do not interfere with encoded, genetic instructions. A weaker surmise is that copy errors among a population of generative replicators are generally more destructive to complexity than reading and development errors. A stronger proposition is that the capacity to increase complexity depends critically and especially on generative replication with low copy errors.

We used a one dimensional linear automaton with a simple selection dynamics. This model produced results that are consistent with our four proposed conditions for a possible generative replicator. If our four proposed conditions are not violated, evolution within the context of the one dimensional automaton will produce more complexity than entropy, indeed approach maximal complexity. The dynamics settles more narrowly around the fitness peak if our four proposed conditions are not violated. The intuition for this result is that generative replication ensures that information flowing into replicators is not detrimental to their survival, even if input signals are noisy.

We have confirmed our complexity conjecture with a wide variety of functional specifications. The results from the selection dynamics reported in the present paper can also be obtained with very different dynamic equations. For example, we have obtained similar results with a specification that used a reinforcement function rather than a selection dynamics. The reinforcement function was a classical habit function (Hull, 1943) where habituation is characterized in terms of an exponentially weighted average of past actions (e.g. Carroll, Overland \& Weil, 2000; Pollak, 1977). The confirmation of our results over a variety of specifications suggests that the complexity conjecture has fairly general validity.

A number of limitations should be considered when our results are extrapolated to realworld cases. First, the linear automaton is obviously a very abstract representation of realworld evolutionary processes, omitting an enormous amount of detail. Second, the entities in our model are extremely simple; they are characterized by one of two possible states. Third, the processes of reading and converting input signals into developmental instructions are represented in a very abstract way. While these limitations caution against hasty extrapolation to real world cases, they are the price paid for using a simple and transparent model that is general enough to capture the essential features of our theoretical argument. What our model offers is a test of our theoretical argument on the basis of a widely accepted stylized representation of evolutionary processes.

The next step is to examine whether the predictions that follow from our argument are met in real world cases. Our findings have wide-reaching implications for the understanding of economic and social evolution. Prior research has eschewed notions of gene-like entities in social evolution because of high error-rates in cultural transmission. Our results suggest that a failure to distinguish between the two kinds of error that are both prominent in social and economic evolution may have led to an erroneous conclusion in that regard. Even if social and economic evolution are very noisy in the sense that evolving entities often misread instructions, complexity can increase if copy error is kept to a minimum.

In the business context, this insight helps understand the puzzling observation that many firms base growth strategies on the idea of creating new units that exactly copy an existing unit. Consider Intel's "Copy Exactly" factory strategy. This strategy solves the problem of getting production facilities up to speed quickly by copying everything at the development 
plant - the process flow, equipment set, suppliers, plumbing, manufacturing clean room, and training methodologies. Everything is selected to meet high volume needs, recorded, and then copied exactly to the high-volume plant. ${ }^{12}$

A theory that can explain the emergence and evolution of such firms as well as other social and economic institutions must distinguish between copy error and read error. According to our complexity conjecture, we would expect that generative replicators that minimize copy error in cultural transmission have been the main drivers of complexity in language, law, markets and other economic institutions. To move from conjecture to facts, we invite empirical research that carefully separates the effects from copy error, on the one hand, and read and development error on the other. The open question is whether enduring differences in error forms will have a systematic effect on levels of expressed complexity in the populations under study.

Further implications relate to prior research on routines. Nelson and Winter (1982) placed the notion of organizational routines at the centre of their evolutionary theory of economic change. Routines have the important virtue for this purpose of being a relatively enduring stable property of the firm and to account, to a significant degree, for variation in firms' performance in product markets. This provides the behavioural foundation for a plausible selection argument in economics (Winter, 1971). Selection becomes operative when the market's signals of profits are correlated with stable operating capabilities.

However, an analytical strength of routines as the building block for an evolutionary theory also poses difficult conceptual challenges when considering the question of generative selection. In prior theory, routines have served the double-duty of both comprising firms' stable operating capabilities (behavioral regularities) and firms' stable latent propensity to manifest such regularity (cognitive regularity). Our definition of routines as generative replicators exposes this problem in prior conceptualizations of routines. More importantly, it also points to replication of routines as a mechanism that plays the role of genetic inheritance in biology.

While it was useful to define routines as observable patterns of interaction among social agents as a first cut (Cohen et al., 1996), it is now time to further refine our understanding. We believe that it is important to define routines as behavioural dispositions (or propensities). They have an observable behavioural component and an unobservable cognitive component among individuals. In order to increase complexity, generative replication of routines must reliably transmit the cognitive component. By contrast, the misreading of instructions leading to mistaken action is not detrimental to the evolution of complexity. Read error will make observable interaction patterns more erratic but does not change the underlying cognitive component of a routine. It is therefore misleading to define routines in terms of observable interaction patterns.

In brief, routines are organizational dispositions to energize conditional patterns of behavior within organizations, involving sequential responses to cues that are partly dependent on social positions in the organization (Hodgson \& Knudsen, in press). Stable patterns of

\footnotetext{
12 See Intel's webpage: http://www.intel.com/pressroom/archive/backgrnd/copy_exactly.htm (consulted 2 April 2008). Other prominent examples of firms that try to stimulate growth by reducing copy error include: McDonalds, Burger King, Pizza Hut, Kentucky Fried Chicken; Holiday Inn, Novotel, Hilton (various brands). Marriott (various brands); Bank of America, Wachovia, HSBC; Merrill Lynch, Starbucks, Cosi; Office Depot, Staples; Borders, Barnes and Noble; Ikea, The Bombay Company; Benetton, Gap. See Knudsen and Winter (unpublished).
} 
interaction are usually a signature of a routine, but at the core of that routine is a cognitive component that maps the reading of cues onto behavior. Even if there is variation in behavior, the cognitive component can be reliably replicated as observed in the gun fire routine (see the introduction).

As noted in the introduction, our argument suggests that we discard any understanding of routines principally in terms of observable patterns of behavior. It is further critical that empirical research on routines does not solely rely on observations of interaction patterns to make inference about the properties of routines. In order to put research on routines back on track, we need carefully designed experiments that allow researchers to make valid inference about the underlying cognitive component of routines. This is not a minor quibble, but rather a condition that must be fulfilled in order to further advance the evolutionary program in the social sciences.

It is also conceivable that generative replication can help solve one of the long-standing puzzles relating to economic and social evolution. How can routines replicate with sufficiently low probability of mutation? Generative replication of routines involves replication of (often simple) cognitive maps among individuals in a group. This map associates the reading of cues with actual behavior. Rather than trying to reduce error in actual behavior, the reduction of error in the functioning of the cognitive map requires much less feedback (less error-correction). Our conjecture therefore implies that economic and social evolution is driven by evolving complexes of cognitive maps within social groups that are replicated over very long periods of time.

\section{References}

Adami, C., 2002. What is complexity? BioEssays 24(12), 1085-1094.

Adami. C., Ofria, C., Collier, T.C., 2000. Evolution of biological complexity. Proceedings of the National Academy of Science 97(9), 4463-4468.

Aunger, R., 2002. The Electric Meme: A New Theory of How We Think. New York: Free Press.

Aarts, H., Dijksterhuis, A., 2000. Habits as Knowledge Structures: Automaticity in GoalDirected Behavior. Journal of Personality and Social Psychology 78, 53-63.

Becker, M.C., (Ed.) 2008. Handbook of Organizational Routines. Cheltenham UK and Northampton MA: Edward Elgar.

Bennett, C.H., 1995. Universal computation and physical dynamics. Physica D: Nonlinear Phenomena 86, Issues 1-2, 268-273.

Blau, J.R., McKinley, W., 1979. Ideas, Complexity, and Innovation. Administrative Science Quarterly 24(2), 200-219.

Brown, S.L., Eisenhardt, K.M., 1997. The Art of Continuous Change: Linking Complexity Theory and Time-Paced Evolution in Relentlessly Shifting Organizations. Administrative Science Quarterly 42(1), 1-34.

Buss, L.W. 1987., The Evolution of Individuality. Princeton, NJ: Gordon and Breach. 
Carroll, C.D., Overland, J., Weil, D.N., 2000. Saving and Growth with Habit Formation. The American Economic Review 90(3), 341-355.

Cohen, M.D., Burkhart, R., Dosi, G., Egidi, M., Marengo, L., Warglien, M., Winter, S.G., 1996. Routines and Other Recurring Action Patterns of Organizations: Contemporary Research Issues. Industrial and Corporate Change 5(3), 653-98.

Davis, B.K., 1979. Complexity Transmission during Replication. Proceedings of the National Academy of Sciences of the United States of America 76(5), 2288-2292.

Davis, B.K., 1994. On Producing More Complexity than Entropy in Replication. Proceedings of the National Academy of Sciences of the United States of America 91(14), 6639-6643.

Dawkins, R., 1976. The Selfish Gene. Oxford: Oxford University Press.

Dawkins, R., 1982. The Extended Phenotype: The Gene as the Unit of Selection. Oxford: Oxford University Press.

Dawkins, R., 1986. The Blind Watchmaker: Why the Evidence of Evolution Reveals a Universe Without Design. New York \& London: W.W. Norton \& Company.

Dawkins, R., 2004. Extended Phenotype - But Not Too Extended. A Reply to Laland, Turner and Jablonka. Biology and Philosophy 19(3), 377-96.

Freitas, R.A., Merkle, R.C., 2004. Kinematic Self-Replicating Machines. Georgetown, TX: Landes Bioscience.

Godfrey-Smith, P., 2000. The Replicator in Retrospect. Biology and Philosophy 15, 403-23.

Gould, S.J. 1996. Life's Grandeur: The Spread of Excellence from Plato to Darwin. London: Cape.

Hodgson, G.M., Knudsen, T., 2004,. The Firm as an Interactor: Firms as Vehicles for Habits and Routines. Journal of Evolutionary Economics 14(3), 281-307.

Hodgson, G.M., Knudsen, T., 2006. Dismantling Lamarckism: Why Descriptions of SocioEconomic Evolution as Lamarckian are Misleading. Journal of Evolutionary Economics, 16(4), 343-66.

Hodgson, G.M., Knudsen, T., 2008. Information, Complexity and Generative Replication. Biology and Philosophy 43(1), 47-65.

Hodgson, G.M., Knudsen, T., (in press) Darwin's Conjecture: The Search for General Principles of Social and Economic Evolution. Chicago: University of Chicago Press.

Hull, C.L. 1943. Principles of Behavior: An Introduction to Behavior Theory. New York: Appleton-Century.

Hull, D.L. 1988. Science as a Process: An Evolutionary Account of the Social and Conceptual Development of Science. Chicago: University of Chicago Press.

Knudsen, T., Winter, S.G. (unpublished). An Evolutionary Model of Spatial Competition. University of Southern Denmark (mimeo).

Luksha, P.O. 2003. Formal Definition of Self-reproductive Systems. In: Standish, R.K., Bedau, M.A., Abbass, H.A. (Eds.) Artificial Life VIII: Proceedings of the Eighth International Conference on Artificial Life. Cambridge, MA: MIT Press, 414-17.

March, J.G., Simon, H.A., 1958. Organizations. New York: Wiley. 
Maynard Smith, J., Szathmáry, E. 1995. The Major Transitions in Evolution. Oxford: WH Freeman Press.

Mayr, E., 1974. Behavior Programs and Evolutionary Strategies. American Scientist 62(6), 650-659.

Mayr, E., 1988. Toward a New Philosophy of Biology: Observations of an Evolutionist. Cambridge, MA and London: Harvard University Press.

McQuiston, D.H. 1989. Novelty, Complexity, and Importance as Causal Determinants of Industrial Buyer Behavior. The Journal of Marketing 53(2), 66-79.

McShea, D.W., 1996. Metazoan complexity and evolution: is there a trend? Evolution 50, 477-492.

Molofsky, J, 1994. Population Dynamics and Pattern Formation in Theoretical Populations. Ecology 1, 30-39.

Morison, E., 1966. Men, Machines, and Modern Times. Cambridge MA: The MIT Press.

Nelson, R.R., Winter, S.G., 1982. An Evolutionary Theory of Economic Change. Cambridge, MA: Harvard University Press.

Pigliucci, M., Kaplan, J., 2006. Making Sense of Evolution: The Conceptual Foundations of Evolutionary Biology. Chicago: University of Chicago Press.

Pollak, R.A., 1977. Habit Formation and Dynamic Demand Functions. The Journal of Political Economy 78(4), 745-763.

Rosser, J.B. Jr. (Ed.) 2009. Handbook of Research on Complexity. Cheltenham UK and Northampton, MA, USA: Edward Elgar.

Shannon, C.E., Weaver, W., 1949. The Mathematical Theory of Communication. Chicago: University of Illinois Press.

Sipper, M., 1998. Fifty Years of Research on Self-Replication: An Overview. Artificial Life 4(3), 237-57.

Sperber, D., 2000. An Objection to the Memetic Approach to Culture. In: Aunger, R. (Ed.) Darwinizing Culture: The Status of Memetics as a Science (Oxford and New York: Oxford University Press), 162-73.

Sterelny, K., Smith, K.C., Dickison, M., 1996. The Extended Replicator. Biology and Philosophy 11, 377-403.

von Neumann, J., 1966. Theory of Self-Reproducing Automata. In: Burks, A. W. (Ed.) Urbana: University of Illinois Press.

Weiss, J.A., 1982. Coping with Complexity: An Experimental Study of Public Policy Decision-Making. Journal of Policy Analysis and Management 2(1), 66-87.

Winter, S.G., 1971. Satisficing, Selection and The Innovating Remnant. The Quarterly Journal of Economics 85(2), 237-261.

Wolfram, S., 1984. Universality and Complexity in Cellular Automata. Physica 10D, 1-35.

Wolfram, S., 2002. A New Kind of Science. Champaign, IL: Wolfram Media, Inc. 